\title{
Experimental Constraints on Left-Right Symmetric Models from Muon Decay
}

\author{
R. Bayes,${ }^{7, *}$ J. F. Bueno, ${ }^{2}$ A. Hillairet, ${ }^{7, *}$ Yu. I. Davydov,${ }^{7,}$ P. Depommier, ${ }^{4}$ W. Faszer, ${ }^{7}$ C. A. Gagliardi, ${ }^{6}$ A. Gaponenko, ${ }^{1, *}$
} D. R. Gill, ${ }^{7}$ A. Grossheim, ${ }^{7}$ P. Gumplinger, ${ }^{7}$ M. D. Hasinoff, ${ }^{2}$ R. S. Henderson, ${ }^{7}$ J. Hu, ${ }^{7,8}$ D. D. Koetke, ${ }^{8}$ R. P. MacDonald, ${ }^{1}$ G. M. Marshall, ${ }^{7}$ E. L. Mathie, ${ }^{5}$ R. E. Mischke, ${ }^{7, \|}$ K. Olchanski, ${ }^{7}$ A. Olin, ${ }^{7, *}$ R. Openshaw, ${ }^{7}$ J.-M. Poutissou, ${ }^{7}$ R. Poutissou, ${ }^{7}$ V. Selivanov, ${ }^{3}$ G. Sheffer, ${ }^{7}$ B. Shin, ${ }^{7, \pi}$ T. D. S. Stanislaus, ${ }^{8}$ R. Tacik, ${ }^{5}$ and R. E. Tribble ${ }^{6}$

\section{(TWIST Collaboration)}

\author{
${ }^{1}$ University of Alberta, Edmonton, Alberta, T6G 2J1, Canada \\ ${ }^{2}$ University of British Columbia, Vancouver, British Columbia, V6T 1Z1, Canada \\ ${ }^{3}$ Kurchatov Institute, Moscow, 123182, Russia \\ ${ }^{4}$ University of Montreal, Montreal, Quebec, H3C 3J7, Canada \\ ${ }^{5}$ University of Regina, Regina, Saskatchewan, S4S OA2, Canada \\ ${ }^{6}$ Texas A\&M University, College Station, Texas 77843, USA \\ ${ }^{7}$ TRIUMF, Vancouver, British Columbia, V6T 2A3, Canada \\ ${ }^{8}$ Valparaiso University, Valparaiso, Indiana 46383, USA \\ (Received 24 October 2010; published 25 January 2011)
}

\begin{abstract}
The TWIST Collaboration has completed a new measurement of the energy-angle spectrum of positrons from the decay of highly polarized muons. A simultaneous measurement of the muon decay parameters $\rho, \delta$, and $\mathcal{P}_{\mu}^{\pi} \xi$ tests the standard model in a purely leptonic process and provides improved limits for relevant extensions to the standard model. Specifically, for the generalized left-right symmetric model $\left|\left(g_{R} / g_{L}\right) \zeta\right|<0.020$ and $\left(g_{L} / g_{R}\right) m_{2}>578 \mathrm{GeV} / c^{2}$, both $90 \%$ C.L.
\end{abstract}

The maximal parity violation of the standard model (SM) charged weak interaction is empirically based. Many natural SM extensions restore parity conservation at a higher mass scale with additional weak couplings. Muon decay is an excellent laboratory to search for these couplings because the purely leptonic process can be calculated very precisely within the SM. This Letter presents a high-precision measurement of the energy-angle spectrum of the positrons emitted in polarized muon decay, which provides new limits for the mass and mixing angle of the heavy $W$ in a class of left-right symmetric models [1].

The most general Lorentz-invariant, derivative-free muon decay matrix element [2] is described by 10 complex, model-independent couplings $\left(g_{\epsilon \mu}^{\gamma}\right)$ involving leftand right-handed leptons ( $\epsilon$ and $\mu$ label the electron and muon, respectively) in scalar, vector, and tensor interactions $(\gamma)$. In the SM, $g_{L L}^{V}=1$, and the other nine constants are zero. When only the positron energy and direction are measured, the muon decay spectrum can be described by four parameters [3], which are bilinear combinations of the $g_{\epsilon \mu}^{\gamma}$ : the Michel parameter $\rho$, as well as $\delta, \mathcal{P}_{\mu} \xi$, and $\eta$. The differential decay rate is then

$$
\begin{aligned}
\frac{d^{2} \Gamma}{d x d(\cos \theta)} \propto & x^{2}\left\{(3-3 x)+\frac{2}{3} \rho(4 x-3)+3 \eta x_{0} \frac{(1-x)}{x}\right. \\
& \left.+\mathcal{P}_{\mu} \xi \cos \theta\left[(1-x)+\frac{2}{3} \delta(4 x-3)\right]\right\},
\end{aligned}
$$

with

$$
\begin{gathered}
x=\frac{E_{e}}{E_{\max }}, \quad x_{0}=\frac{m_{e}}{E_{\max }}, \quad \mathcal{P}_{\mu}=\left|\overrightarrow{\mathcal{P}}_{\mu}\right|, \\
\cos \theta=\frac{\overrightarrow{\mathcal{P}}_{\mu} \cdot \vec{p}_{e}}{\left|\overrightarrow{\mathcal{P}}_{\mu}\right|\left|\vec{p}_{e}\right|} .
\end{gathered}
$$

The neutrino masses are neglected; $E_{\max }=52.828 \mathrm{MeV}$. Radiative corrections [4] are not explicitly shown but are significant and must be evaluated within the SM to a precision comparable to the experiment. The polarization of the muon from pion decay begins as $\mathcal{P}_{\mu}^{\pi}$ and may evolve over the $2.2 \mu$ s mean lifetime of the muon to become $\mathcal{P}_{\mu}$ at the time of decay. The SM predictions are $\rho=\delta=3 / 4$, $\mathcal{P}_{\mu}^{\pi}=\xi=1$, and $\eta=0$. Precision measurements of these parameters test the SM predictions and are sensitive to extensions to the SM.

Prior to the TRIUMF weak interaction symmetry test (TWIST) experiment, $\rho, \delta$, and $\mathcal{P}_{\mu}^{\pi} \xi$ were known with uncertainties in the range of $(3.5-8.5) \times 10^{-3}$ [5]. Intermediate TWIST results have already reduced those uncertainties to $(0.7-3.8) \times 10^{-3}$ [6,7]. TWIST has now realized its goal of making about an order of magnitude improvement in each of the parameters. These final results of the experiment supersede those in our previous publications.

TWIST used highly polarized positive muons from pions decaying on the surface of a graphite production target irradiated with $500 \mathrm{MeV}$ protons. The muons were 
transported by the TRIUMF M13 surface muon channel to the entrance of a $2 \mathrm{~T}$ superconducting solenoidal magnet and were guided by the field along its symmetry axis into the detector array [8]. A thin $(239 \mu \mathrm{m})$ trigger scintillator identified muons entering the detector. The muons were ranged to stop predominately in a thin metal foil at the center of a symmetric stack of high-precision, low-mass planar multiwire chambers. There were 6 proportional and 22 drift chambers, surrounded by helium gas, on each side of the stopping target. Ionization of tracks from decay positrons was sampled by the chambers, and drift times were recorded by time-to-digital converters.

With a muon rate of $(2-5) \times 10^{3} \mathrm{~s}^{-1}$, data sets of $10^{9}$ events could be obtained within a few days. Data sets were taken with two foil stopping targets: Ag (30.9 $\mu \mathrm{m}$ thick) and $\mathrm{Al}(71.6 \mu \mathrm{m}$ thick), each $>99.999 \%$ pure. Sets were also taken with deliberately altered conditions to assist in studies of possible systematic errors. A pair of time expansion chambers [9] was inserted upstream of the solenoid to determine the incoming muon beam characteristics. Because they caused multiple scattering and hence muon depolarization, the time expansion chambers were removed during most data taking. This phase of the experiment was completed in 2007.

Analyzed events were collected into two-dimensional (2D) distributions of positron angle and momentum (or energy) whose shape depends on the decay parameters. These distributions from data were compared to similar ones derived from a GEANT3 simulation [10]. Both were subjected to essentially the same analysis, allowing biases and inefficiencies to be included in an equivalent way to reduce the dependence of the result on the specific analysis procedure. This places great importance on the accuracy and detail of the simulation, which includes not only standard physics processes but also a detailed description of the beam, magnetic field, geometry, and detector response. Decay parameters were extracted by fitting the 2D data distribution to that of a base distribution of simulated events, plus simulated distributions corresponding to the first derivatives of the spectrum with respect to decay parameters (or combinations thereof), yielding fit coefficients $\Delta \rho, \Delta \xi$, and $\Delta \xi \delta$. The decay parameters used in the generation of the simulation were hidden, so the analysis was "blind" [6].

Fourteen data sets were used to extract $\rho$ and $\delta$, seven with each of the $\mathrm{Ag}$ and $\mathrm{Al}$ targets. Only nine sets were used for $\mathcal{P}_{\mu}^{\pi} \xi$; the other five were acquired to test consistency and systematic effects with altered beam, magnetic field, or muon multiple scattering, where the depolarization was not optimally controlled. The residuals for the fit of one nominal data set in units of standard deviations $(\sigma)$ are shown in Fig. 1. A histogram of the residuals in the fiducial region, summed over all sets, has a mean of $-0.003 \pm$ 0.005 and $\sigma=1.002 \pm 0.004$. Also shown is the range of $(p, \cos \theta)$ used to determine the decay parameters. The fiducial cuts are symmetric for upstream and downstream

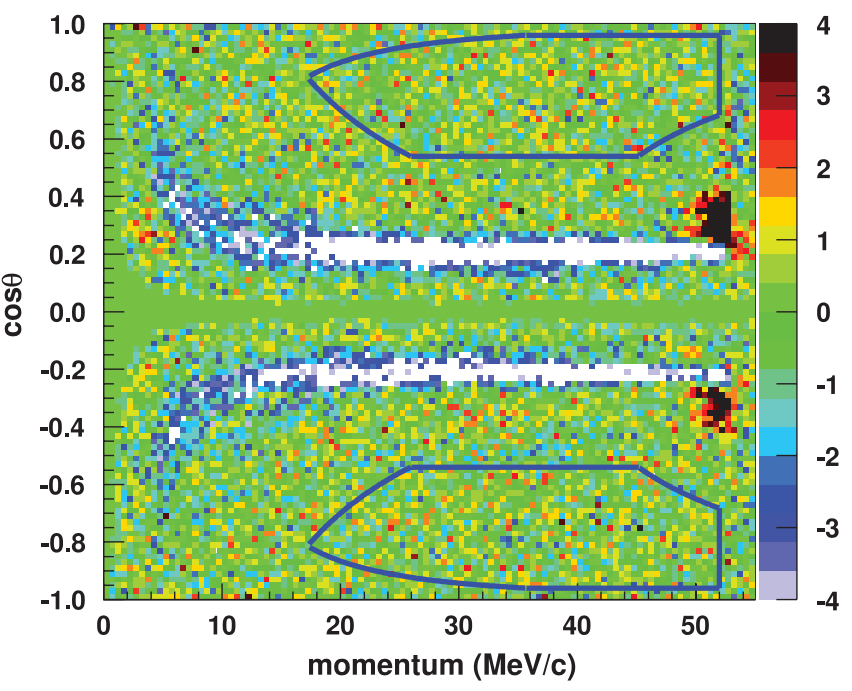

FIG. 1 (color). Residuals for the fit of one nominal data set to simulation in units of standard deviations. The fiducial region is outlined.

decays and were selected to maximize sensitivity to the decay parameters while reducing systematic uncertainties. For all 14 data sets, there were $11 \times 10^{9}$ events, of which $0.55 \times 10^{9}$ passed all event selection criteria and were within the fiducial region. Simulation sets were typically 2.7 times larger than the corresponding data set. The consistency of the data sets (statistical uncertainties only) was assessed by fits to constant means for the values of $\Delta \rho, \Delta \delta$, and $\Delta \mathcal{P}_{\mu}^{\pi} \xi$, which gave reduced $\chi^{2}$ values of $14.0 / 13$, $17.7 / 13$, and $9.7 / 8$, respectively.

The procedure of fitting the difference of two spectra in terms of derivatives also provides a natural tool for the evaluation of systematic uncertainties. The simulation was validated through comparison with observables in the data that do not depend on muon decay parameter values, and the resulting uncertainties were factored into estimates of the systematic uncertainties. The sensitivities were obtained from the effect on the decay parameters when an identified source of systematic uncertainty was changed (often by an exaggerated amount) in one of the spectra. This was typically achieved with two simulated spectra. The systematic uncertainties are listed, along with the statistical errors, in Table I.

Notable improvements in the systematic uncertainties for $\rho$ and $\delta$ compared to our intermediate results [6] were achieved for positron interactions, chamber response, and momentum calibration. The positron interactions systematic addresses the possible inaccuracy in the simulation of reproducing positron energy loss in the stopping target and detector elements, primarily due to bremsstrahlung, deltaray production, and ionization. It was better constrained by comparisons of identified interactions observed in the data and in the simulation. Chamber response refers to the conversion of drift times to spatial information used in track fitting to evaluate the momentum and angle of each decay positron. This was improved by more precise 
TABLE I. Systematic uncertainties and statistical errors for $\rho$, $\delta$, and $\mathcal{P}_{\mu}^{\pi} \xi$.

\begin{tabular}{|c|c|c|c|}
\hline Uncertainties & $\begin{array}{c}\rho \\
\left(\times 10^{-4}\right)(\times\end{array}$ & $\begin{array}{l}\delta \\
\left.10^{-4}\right)\end{array}$ & $\begin{array}{c}\mathcal{P}_{\mu}^{\pi} \xi \\
\left(\times 10^{-4}\right) \\
\end{array}$ \\
\hline \multicolumn{4}{|l|}{ Target-independent systematics } \\
\hline Momentum calibration & 1.2 & 1.2 & 1.5 \\
\hline Chamber response & 1.0 & 1.8 & 2.3 \\
\hline Radiative corrections, $\eta$ & 1.3 & 0.6 & 1.2 \\
\hline Resolution & 0.6 & 0.7 & 1.5 \\
\hline Positron interactions ${ }^{\mathrm{a}}$ & 0.5 & 0.2 & 0.4 \\
\hline Others & 0.3 & 0.3 & 0.4 \\
\hline Depolarization in fringe field & & & $+15.8,-4.0$ \\
\hline Depolarization in stopping target & & & 3.2 \\
\hline$\pi$ decays in beam line & & & 1.0 \\
\hline \multicolumn{4}{|l|}{ Uncertainties for $\mathrm{Ag}$ target } \\
\hline Bremsstrahlung rate & 1.8 & 1.6 & 0.5 \\
\hline Ag thickness/stop position & 3.8 & 6.4 & 0.6 \\
\hline Statistical & 1.2 & 2.1 & 4.2 \\
\hline \multicolumn{4}{|l|}{ Uncertainties for $\mathrm{Al}$ target } \\
\hline Bremsstrahlung rate & 0.7 & 0.7 & 0.3 \\
\hline Al thickness/stop position & 0.2 & 0.8 & 0.8 \\
\hline Statistical & 1.4 & 2.4 & 3.9 \\
\hline Weighted systematic & 2.3 & 2.7 & $+16.5-6.3$ \\
\hline Weighted statistical & 1.2 & 2.1 & 2.9 \\
\hline Total error & 2.6 & 3.4 & $+16.8-6.9$ \\
\hline
\end{tabular}

${ }^{a}$ Excluding bremsstrahlung.

monitoring and control of atmospheric influences that could change the chamber cell geometry. In addition, a method was devised [11] to calibrate the chambers' spacetime relations, for each plane, in both data and simulation, thereby reducing reconstruction biases. The maximum positron energy provides a calibration feature that was used to reduce the energy scale uncertainty. Since energy loss varies with the track angle linearly in $1 /(\cos \theta)$ due to the planar geometry of the detector, the region near the kinematic end point of $52.8 \mathrm{MeV} / c$ was matched for data and simulation for small bins of $\cos \theta$. The data-simulation relative energy calibration procedure has undergone improvements to become more robust to fitting conditions.

The asymmetry parameter $\xi$ is also subject to uncertainties from these sources, but they are overshadowed by uncertainties unique to depolarization, as shown in Table I. Depolarization in the fringe field and in the muon stopping target result in $\mathcal{P}_{\mu}<\mathcal{P}_{\mu}^{\pi}$ and constitute the largest contributions to systematic uncertainties for $\mathcal{P}_{\mu}^{\pi} \xi$. These uncertainties were improved considerably for this analysis compared to the intermediate result [7]. Improvements in the beam steering reduced the fringe field depolarization for a nominal data set to only $2.5 \times 10^{-3}$. The uncertainty depends on the accuracy of simulating the muon spin evolution as the beam passes through significant radial field components at the solenoid entrance. The essential ingredients are an accurate field map and precise knowledge of the position and direction of the muons, as provided by the time expansion chambers. Depolarization in the stopping target from muon spin relaxation is assessed from the measured time dependence of the asymmetry.

After revealing the hidden parameters, the results for the three decay parameters are consistent with the SM predictions. While the generalized matrix element treatment of Ref. [2] does not constrain the sign of deviations from the SM values for $\rho, \delta$, and $\xi$, the product $\mathcal{P}_{\mu}^{\pi} \xi \delta / \rho$ is constrained to be $\leq 1.0$ and is 1.0 in the SM. This quantity defines the asymmetry between $\cos \theta= \pm 1$ at the maximum decay positron energy. Our decay parameter values combined to give $\mathcal{P}_{\mu}^{\pi} \xi \delta / \rho=1.00192_{-0.00066}^{+0.00167}$ (the errors account for significant correlations), and the initial evaluation of $\mathcal{P}_{\mu}^{\pi} \xi \delta / \rho$ showed that the value for the Ag data was higher than that for $\mathrm{Al}$ by $3.8 \sigma$. This apparent contradiction initiated an exhaustive reconsideration of effects that might have been overlooked in the blind analysis. The review showed that effects such as $\mu^{+} \rightarrow e^{+} X^{0}$ decays (where $X^{0}$ is a long-lived unobserved particle), an incorrect value of the $\eta$ parameter, or plausible errors in the radiative correction implementation were not responsible for the unexpected $\mathcal{P}_{\mu}^{\pi} \xi \delta / \rho$ value.

While no obvious mistake was uncovered in the estimates of the systematic uncertainties previously considered, we found that two corrections had been missed. A small correction was added for muon radiative decay $\left(<1 \times 10^{-4}\right.$ for the $\mathrm{Ag}$ data and negligible for $\left.\mathrm{Al}\right)$. Another correction was made for each set to account for a difference between the mean muon stopping position for data and simulation. We also concluded that the uncertainties for the two targets were sufficiently different to merit dividing the systematic uncertainties into common and target-dependent categories. The target-independent systematics are unchanged from the blind analysis. Separate uncertainties for bremsstrahlung were computed, and an additional sensitivity to the muon stopping position in the target was added.

With these changes the central values of $\rho$ and $\delta$ decreased from the blind results by 0.00014 and 0.00023 , respectively. $\mathcal{P}_{\mu}^{\pi} \xi$ is unchanged and its error reduced after including information from the measurement of $\delta$ in the five sets not used for $\mathcal{P}_{\mu}^{\pi} \xi$. All uncertainties changed by $<0.00006$. The difference between targets for $\mathcal{P}_{\mu}^{\pi} \xi \delta / \rho$ is reduced to $\sim 1 \sigma$, and $\mathcal{P}_{\mu}^{\pi} \xi \delta / \rho=1.00179_{-0.00071}^{+0.00156}$. The revised results are compared to prior results in Fig. 2. The values, including the uncertainties from Table I, are

$$
\begin{aligned}
\rho & =0.74977 \pm 0.00012 \text { (stat) } \pm 0.00023 \text { (syst) } \\
\delta & =0.75049 \pm 0.00021(\text { stat }) \pm 0.00027 \text { (syst) } \\
\mathcal{P}_{\mu}^{\pi} \xi & =1.00084 \pm 0.00029(\text { stat })_{-0.00063}^{+0.00165} \text { (syst). }
\end{aligned}
$$

The decay parameters measured by TWIST contribute to a larger set derived from other muon decay observables that can be analyzed in terms of the weak couplings $g_{\epsilon \mu}^{\gamma}$. A global analysis $[6,12]$ imposes $\mathcal{P}_{\mu}^{\pi} \xi \delta / \rho \leq 1.0$ and yields $\mathcal{P}_{\mu}^{\pi} \xi \delta / \rho>0.99909$ (90\% C.L.), compared to the pre-TWIST lower limit $\mathcal{P}_{\mu}^{\pi} \xi \delta / \rho>0.99682$ (90\% C.L.) 


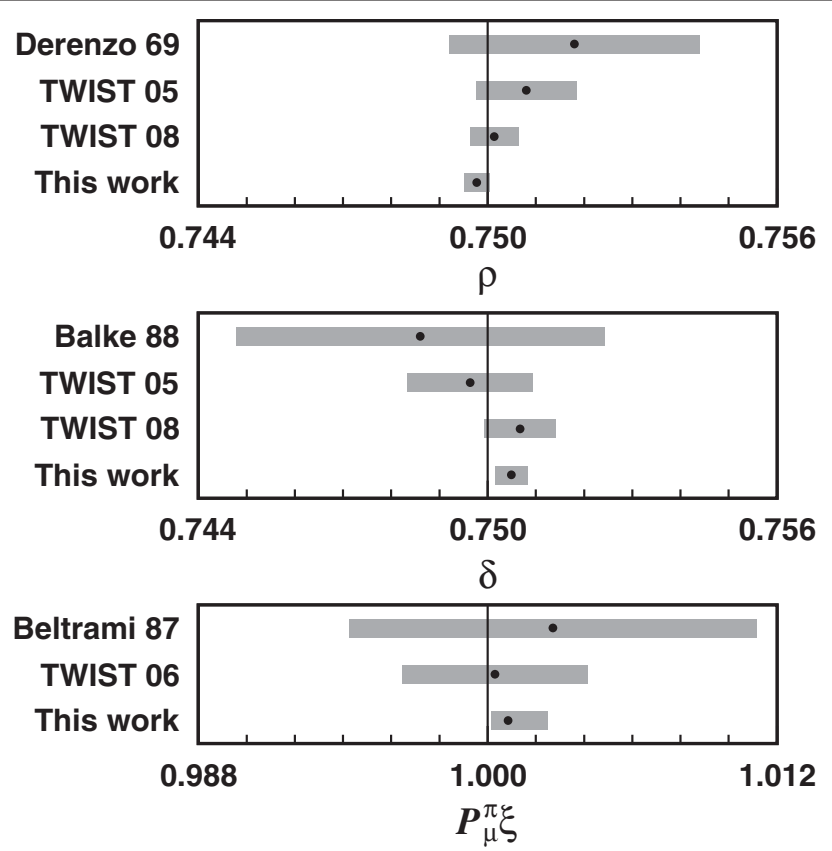

FIG. 2. Summary of published central values and total uncertainties for $\rho, \delta$, and $\mathcal{P}_{\mu}^{\pi} \xi[5-7,14,15]$, along with the results of this analysis. Vertical lines represent the SM values.

[13]. The global analysis confirms consistency with the SM, where $g_{L L}^{V}$ is the only nonzero term. The TWIST results restrict the upper limits of other terms. For example, the limit on the total right-handed muon coupling

$$
Q_{R}^{\mu}=\frac{1}{4}\left|g_{L R}^{S}\right|^{2}+\frac{1}{4}\left|g_{R R}^{S}\right|^{2}+\left|g_{L R}^{V}\right|^{2}+\left|g_{R R}^{V}\right|^{2}+3\left|g_{L R}^{T}\right|^{2}
$$

is reduced by a factor of 6 from the pre-TWIST value to $<8.2 \times 10^{-4}$ (90\% C.L.).

Left-right symmetric models extend the SM with a righthanded $W$ [1]. In the generalized (or nonmanifest) model no assumptions are made about the ratio of right- to lefthanded couplings $\left(g_{R} / g_{L}\right)$ or the form of the right-handed Cabibbo-Kobayashi-Maskawa matrix. In this case, the TWIST result for $\rho$ provides the best limit on the mixing angle between the light and heavy mass eigenstates $W_{1}$ and $W_{2}$. Our limit is $\left|\left(g_{R} / g_{L}\right) \zeta\right|<0.020$ (90\% C.L.), compared to the pre-TWIST limit of $\left|\left(g_{R} / g_{L}\right) \zeta\right|<0.066$. The lower limit on the mass of $W_{2}\left[\left(g_{L} / g_{R}\right) m_{2}\right]$ has been increased from 400 to $578 \mathrm{GeV} / c^{2}$. Coupled constraints on the mass for $\left(g_{L} / g_{R}\right) m_{2}$ and the mixing angle are shown in Fig. 3, where our limits are derived from a correlated 2D probability distribution from our measurements. These improved constraints will significantly impact predictions from the class of left-right symmetric models where the neutrinos are light compared to the muon mass.

We thank all early TWIST collaborators and students for their substantial contributions, as well as C. Ballard, M. Goyette, and the TRIUMF cyclotron operations, beam lines, and support personnel. Computing resources were provided by WestGrid and Compute/Calcul Canada. This work was supported in part by the Natural Sciences and

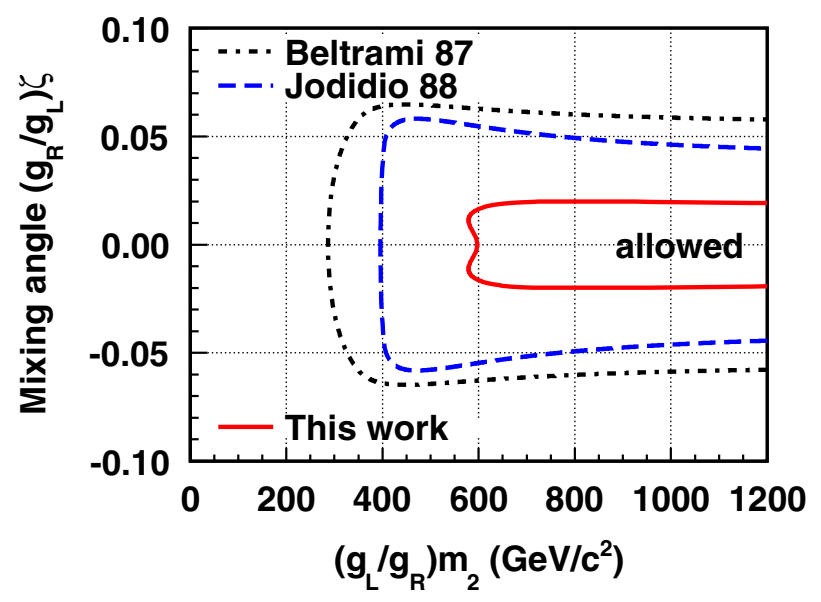

FIG. 3 (color online). Allowed region (90\% C.L.) of mixing angle $(\zeta)$ and heavy $W$ mass $\left(m_{2}\right)$ for the general left-right symmetric model.

Engineering Research Council and the National Research Council of Canada, the Russian Ministry of Science, and the U.S. Department of Energy.

\footnotetext{
*Also at University of Victoria, Victoria, BC, Canada.

†Present address: JINR, Dubna, Russia.

${ }^{\ddagger}$ Present address: LBNL, Berkeley, CA, USA.

${ }^{\S}$ Present address: AECL, Mississauga, ON, Canada.

"mischke@triumf.ca

"Also at University of Saskatchewan, Saskatoon, SK, Canada.
}

[1] P. Herczeg, Phys. Rev. D 34, 3449 (1986).

[2] W. Fetscher, H-J. Gerber, and K. F. Johnson, Phys. Lett. B 173, 102 (1986); see review in K. Nakamura et al. (Particle Data Group), J. Phys. G 37, 075021 (2010).

[3] L. Michel, Proc. Phys. Soc. London Sect. A 63, 514 (1950); C. Bouchiat and L. Michel, Phys. Rev. 106, 170 (1957); T. Kinoshita and A. Sirlin, Phys. Rev. 108, 844 (1957).

[4] A. Arbuzov and K. Melnikov, Phys. Rev. D 66, 093003 (2002), and references therein.

[5] S. E. Derenzo, Phys. Rev. 181, 1854 (1969); I. Beltrami et al., Phys. Lett. B 194, 326 (1987); B. Balke et al., Phys. Rev. D 37, 587 (1988).

[6] R. P. MacDonald et al., Phys. Rev. D 78, 032010 (2008).

[7] B. Jamieson et al., Phys. Rev. D 74, 072007 (2006).

[8] R. S. Henderson et al., Nucl. Instrum. Methods Phys. Res., Sect. A 548, 306 (2005).

[9] J. Hu et al., Nucl. Instrum. Methods Phys. Res., Sect. A 566, 563 (2006).

[10] R. Brun et al., GEANT3 Users Guide, CERN Program Library W5013, 1994.

[11] A. Grossheim et al., Nucl. Instrum. Methods Phys. Res., Sect. A 623, 954 (2010).

[12] C. A. Gagliardi, R. E. Tribble, and N. J. Williams, Phys. Rev. D 72, 073002 (2005).

[13] A. Jodidio et al., Phys. Rev. D 34, 1967 (1986); 37, 237(E) (1988).

[14] J. R. Musser et al., Phys. Rev. Lett. 94, 101805 (2005).

[15] A. Gaponenko et al., Phys. Rev. D 71, 071101(R) (2005). 